\title{
¿Ocurrió efecto contagio en los mercados de acciones de América Latina durante la crisis financiera global?
}

\author{
DE JESÚS GUTIÉRREZ, RAÚL \\ Facultad de Economía \\ Universidad Autónoma del Estado de México \\ Correo electrónico: rjg2005mx@yahoo.com.mx
}

\begin{abstract}
RESUMEN
Este trabajo prueba la existencia de contagio financiero entre los mercados de acciones de la región de América Latina y el mercado de acciones de Estados Unidos basado en el análisis del comportamiento de las correlaciones en periodos de estabilidad y crisis. El estudio emplea un modelo GARCH de correlaciones condicionales dinámicas multivariado para estimar las correlaciones cambiantes en el tiempo, y utiliza la prueba estadística-t bajo un procedimiento bootstrap para analizar los posibles canales de efectos de contagio financiero en los mercados de acciones emergentes. Los resultados muestran que las correlaciones estimadas se incrementaron en el periodo de la turbulencia financiera, como consecuencia de la presencia de cambios estructurales fuertes. Asimismo, el estudio proporciona evidencia de que los mercados de acciones de Brasil, Chile, Colombia, México y Perú son fuertemente contagiados durante la crisis financiera global. Sin embargo, el mercado de acciones de Argentina muestra evidencia de interdependencia con respecto al mercado de acciones de Estados Unidos. Los hallazgos tienen importantes implicaciones para los inversionistas y diseñadores de la política económica que buscan apropiados mecanismos para evitar los efectos negativos del contagio financiero en los mercados de acciones emergentes.
\end{abstract}

Palabras clave: mercados de acciones emergentes, contagio financiero, crisis financiera global, modelos MGARCH-CCD.

Clasificación JEL: C5; C22; G14; G15.

MSC2010: 62F40; 62M10; 91B54; 91G70.

Artículo recibido el 15 de mayo de 2018 y aceptado el 14 de mayo de 2019. 


\title{
Did the contagion effect occur on the Latin America stock markets during the global financial crisis?
}

\begin{abstract}
This paper tests the existence of financial contagion between US and Latin America stock markets based on the analysis of pattern of the correlation coefficients during crisis and stable periods. The study applies a dynamic conditional correlation multivariate GARCH model to estimate time-varying correlations and adopts the t-statistics test under a bootstrap procedure to examine the potential channels of financial contagion effects on emerging stock markets. The empirical results confirm that the estimated correlations has increased significantly in the period of financial turmoil as result of the presence of strong structural changes. Moreover, the study provides evidence that Brazil, Chile, Colombia Mexico and Peru stock markets are significantly affected by the contagion effects from the global financial crisis. However, Argentina stock market exhibits strong evidence of interdependence with the USA stock market. The findings have important implications for investors and policy makers, which seek preventive mechanisms to avoid negative effects of the financial contagion in emerging stock markets.
\end{abstract}

Keywords: emerging stock markets, contagion, global financial crisis, DCC-MGARCH models.

JEL classification: C5; C22; G14; G15.

MSC2010: 62F40; 62M10; 91B54; 91G70.

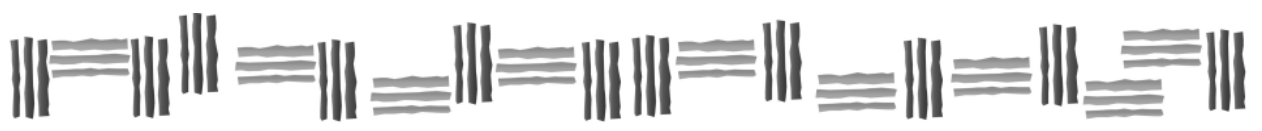




\section{Introducción.}

El nuevo milenio ha sido testigo de varias crisis económicas y financieras, que han provocado importantes desequilibrios y efectos devastadores en gobiernos, bancos centrales y mercados financieros de países desarrollados y emergentes. En este contexto, la crisis financiera global, iniciada con el estallido de la burbuja inmobiliaria y la quiebra del banco Lehman Brothers, ha sido considerada el peor desastre financiero desde la Gran Depresión de 1929. La magnitud de la crisis generó incertidumbre entre instituciones financieras e inversionistas y deterioró la imagen de las autoridades regulatorias financieras, lo que incrementó inmediatamente el nivel de la volatilidad en los mercados financieros de Estados Unidos, América Latina, Europa y Asia. En consecuencia, la capitalización de los mercados de acciones se desplomó en forma paralela por la falta de liquidez y el pánico financiero. La mayoría de las monedas de la región de América Latina se depreciaron frente al dólar estadounidense y al euro, lo que generó catastróficas pérdidas en los balances de las empresas debido a operaciones cambiarias y de coberturas de riesgos, particularmente en Brasil, Chile, México y Perú.

Asimismo, la restricción y el encarecimiento del crédito se agudizó en el sector bancario. Este hecho se vio reflejado en el desempeño de numerosas empresas, que requieren de financiación interna y externa para emprender nuevos proyectos de inversión y planificar oportunamente sus gastos e ingresos. De esta manera, los efectos negativos de la crisis y su rápida transmisión hacia otros sistemas financieros internacionales, originó un efecto de contagio a nivel global sin una justificación fundamental económica. En la literatura financiera reciente, la discusión sobre el fenómeno de contagio continúa llamando la atención de los académicos, inversionistas institucionales, diseñadores de la política económica y reguladores. Aunque no existe un consenso sobre una definición correcta en la literatura; sin embargo, los diferentes conceptos utilizados difieren con la naturaleza específica de los estudios.

En este tenor, diferentes técnicas cuantitativas han sido adoptadas para el análisis del fenómeno de contagio que van desde modelos de cointegración lineal y no-lineal, modelos de correlaciones dinámicas hasta aproximaciones de onda corta (wavelets) (Baur, 2003; Chiang, Jeon \& Li, 2007; Corsetti, Pericoli \& Sbracia, 2005; Gallegati, 2012). De acuerdo con Forbes y Rigobon (2002), el contagio se define como el incremento de las correlaciones entre mercados financieros en periodos de crisis o extrema volatilidad. En contraste, otros autores definen al contagio como la transmisión de choques internos y externos entre mercados. Este mecanismo de propagación es generado por importantes variaciones en indicadores macroeconómicos claves, así como por el comportamiento de los inversionistas en los mercados financieros emergentes durante periodos de crisis.

Sin embargo, la propagación de choques de un mercado a otro es difícil de explicar como consecuencia de la ardua tarea para encontrar excelentes indicadores que coadyuven en la evaluación del impacto de los fundamentos macroeconómicos. En consecuencia, una forma más sencilla de estudiar este fenómeno es a través del análisis de la evolución de las correlaciones entre dos mercados financieros durante periodos de estabilidad (pre-crisis) y turbulencia financiera (crisis). De acuerdo con el trabajo de Celik (2012), si dos mercados financieros se encuentran ligeramente correlacionados durante un periodo estable y la presencia de un choque en un mercado incrementa las correlaciones considerablemente, este hecho es evidencia de contagio entre mercados financieros. Por otra parte, cuando dos mercados financieros comúnmente están altamente correlacionados y después de un choque continúan con la misma tendencia, este fenómeno se conoce en la literatura como interdependencia, que se deriva de los estrechos vínculos reales entre dos economías.

El estudio de la naturaleza del contagio en episodios de alta volatilidad en los mercados financieros internacionales tiene importantes implicaciones en la economía global y en las finanzas. Particularmente en las áreas relacionadas con la diversificación internacional y eficiente asignación de capital, la formulación de la política monetaria, regulación financiera, política fiscal, valoración de activos financieros, integración de los mercados financieros, administración del riesgo y diseño de estrategias de cobertura. Por ello, el objetivo del trabajo es probar la existencia de contagio financiero entre seis mercados de acciones de América Latina y el mercado de acciones de Estados Unidos en el 
periodo del 2 de enero de 2000 al 31 de diciembre de 2009. La pregunta que se pretende responder es la siguiente: ¿Los mercados de acciones de Argentina, Brasil, Chile, Colombia, México, Perú evitaron el efecto devastador de la crisis financiera global?

El estudio contribuye a la literatura sobre contagio financiero en varios aspectos. En primer lugar, el trabajo estudia el tema del efecto contagio en un grupo de mercados de acciones de la región de América Latina: Argentina, Brasil, Chile, Colombia, México y Perú. En este sentido, es importante destacar que el estudio utiliza los precios diarios de los índices de las bolsas de valores a diferencia de Arouri, Lahiani y Nguyen (2013), quienes usan datos mensuales de los índices de Morgan Stanley Capital International (MSCI, por sus siglas en inglés). El análisis también se extiende para incluir a los mercados de acciones de Colombia y Perú, países que no habían sido considerados en la literatura previa y reciente. En segundo lugar, el estudio aplica un modelo GARCH de correlaciones condicionales dinámicas multivariado (MGARCH-CCD) para analizar las relaciones de dependencia entre el mercado de acciones de Estados Unidos y los mercados de acciones de Argentina, Brasil, Chile, Colombia, México y Perú en el contexto de la crisis financiera global. La estructura GARCH multivariada permite estimar las correlaciones condicionales dinámicas (CCD) fuera de la muestra en periodos de estabilidad y crisis financiera. El uso de las CCD fuera de la muestra es importante para el análisis de posibles canales de efectos de contagio financiero, puesto que captura el efecto del día de la semana y otros eventos. Un hecho bien documentado es que los inversionistas en los mercados de acciones reaccionan de forma instantánea a la llegada de nueva información, debido a que afecta las varianzas y el patrón de las CCD en diferentes niveles a diferencia de las CCD dentro de la muestra utilizadas por Arouri et al. (2013). Este hecho permitirá capturar el fenómeno de la heterocedasticidad condicional y evitar correlaciones sesgadas como resultado de las variaciones en los periodos de baja y alta volatilidad. En este marco, Forbes y Rigobon (2002) corrigen el sesgo de las correlaciones como una alternativa para medir el contagio financiero. Sin embargo, la evidencia empírica revela que el contagio pocas veces ocurre en los periodos de crisis financiera.

De esta manera, la naturaleza cambiante en el tiempo de las CCD similar al de un proceso no estacionario, proporciona más información para explicar el contagio financiero que las características de las fluctuaciones de las series financieras, que comúnmente son utilizadas en el análisis de cointegración y modelo de vectores de corrección de error. Aunque es importante mencionar que el modelo MGARCH-CCD no captura totalmente la no-linealidad y los diferentes niveles de asimetría observados en las series financieras como el método de cópulas. Aunque el análisis de la estructura de dependencia es aún estático, por lo que también requiere de la filtración de los rendimientos a través de modelos GARCH para explicar apropiadamente el fenómeno de contagio en el contexto de nolinealidad-condicional.

En términos de validación y confiabilidad, el estudio también contribuye a la existencia de contagio financiero, al adoptar el estadístico $t$ bajo un procedimiento de replicación bootstrap de las CCD fuera de la muestra para determinar el valor- $p$ de la prueba de la diferencia en las medias. La última contribución del estudio consiste en la exploración de la naturaleza cambiante en el tiempo de las relaciones entre la volatilidad condicional y las correlaciones condicionales a través de un análisis de regresión por cuantiles. Este análisis es importante porque si la tendencia de las correlaciones y volatilidad presentan el mismo comportamiento, propiciará que el impacto del riesgo sea más severo en el largo plazo que en el corto plazo, particularmente durante periodos de extrema volatilidad. De hecho, este análisis proporciona nueva evidencia robusta de que el contagio financiero se transmitió desde el mercado de acciones de Estados Unidos hacia los 6 mercados de acciones de América Latina durante la intensificación de la crisis financiera global.

\section{Revisión de la literatura.}

Una gran cantidad de autores han estudiado el efecto de contagio en diferentes contextos de crisis financieras y mercados financieros de diversos países. En un estudio sobre países emergentes, Mathur, Gleason y Singh (2002) proporcionan evidencia de efectos de contagio de la crisis del Peso Mexicano 
de 1994, que fueron trasmitidos de manera muy eficaz del mercado de acciones mexicano hacia el mercado de acciones chileno. Utilizando las correlaciones ajustadas por heterocedasticidad para diferentes países de Europa, Serwa y Bohl (2005) determinan inestabilidad en las relaciones de dependencia después de la crisis. Además, los mercados de acciones de Europa central y oriental no son más vulnerables al efecto de contagio de la crisis financiera asiática de 1997 que los mercados de acciones de Europa occidental. Rodriguez (2007) modela la relación de dependencia a través de cópulas dinámicas para 5 y 4 mercados de acciones de las regiones de Asia y América Latina. Los hallazgos revelan evidencia de contagio debido a que las correlaciones cambian durante los periodos de las crisis asiática de 1997 y mexicana de 1994. Asimismo, los estudios de Cho y Parhizgari (2008), Khan y Park (2009) sustentan la presencia de contagio en los mercados de acciones de Corea, Hong Kong, Taiwán y Singapur, y su efecto se extiende más tarde hacia Malasia, Tailandia, Indonesia y Filipinas.

Horta, Mendes y Vieira (2009) utilizan la teoría de cópulas para probar la existencia de contagio de la crisis inmobiliaria de EEUU hacia los mercados de acciones de Europa en el periodo 2005-2008. No obstante, los resultados confirman la presencia de efectos de contagio para los países de Canadá, Francia, Italia, Reino Unido, excepto Alemania. Naoui, Liouane y Brahim (2010) encuentran fuerte evidencia de contagio durante la crisis financiera global entre el mercado de acciones de EEUU y mercados de acciones desarrollados y emergentes, estimando un modelo GARCH multivariado de correlaciones condicionales dinámicas. De la misma manera, Syllignakis y Kouretas (2011) analizan la estructura de correlación de los rendimientos de 7 mercados de acciones de Europa central y oriental en el periodo 1997-2009. Los resultados revelan que los mercados de acciones emergentes están expuestos a choques externos con cambio de régimen en las correlaciones condicionales. Inci, Li y McCarthy (2011) aplican el análisis de correlación local para estudiar el contagio en índices de acciones desarrollados. Sus hallazgos confirman la presencia de contagio del S\&P500 hacia el DAX de Alemania, FTSE 100 de Londres, Nikkei 225 de Japón y Hang Seng de Hong Kong en el periodo del 3 de enero de 1985 al 8 de noviembre de 2008.

Por su parte, Bouaziz, Selmi y Boujelbene (2012) adoptan un modelo GARCH con cambio de régimen de Markov, y demuestran la presencia del efecto contagio del índice S\&P500 sobre los índices de acciones CAC 40 de Francia, DAX de Alemania, MIB de Italia y FTSE 100 de Reino Unido durante la crisis financiera global. En un estudio que utiliza aproximaciones de ondas corta para los países del bloque G7, Brasil y Hong Kong, Gallegati (2012) proporciona evidencia concluyente de que todos los mercados de acciones analizados han sido afectados por la crisis subprime de EEUU, particularmente Brasil y Japón. Horvath y Poldaufy (2012) estudian el fenómeno de contagio a través de los modelos GARCH VECH y BEKK, y encuentran evidencia de que la crisis financiera global representó un choque común para los mercados de acciones de Alemania, Australia, Brasil, Canadá, China, Hong Kong, Japón, Rusia, Sudáfrica, Reino Unido y EEUU debido a que las correlaciones se incrementaron ligeramente. Asimismo, Peng y $\mathrm{Ng}$ (2012) estudian la relación de dependencia entre 5 índices de acciones internacionales y sus correspondientes índices de volatilidad con una aproximación de cópula dinámica. Los hallazgos indican la existencia de contagio financiero y asimetría en el coeficiente de dependencia de la cola. Aunque algunas veces el contagio no puede ser detectado de manera clara por los movimientos de los índices de acciones, sino por la dependencia entre los índices de volatilidad.

Otro estudio más reciente que ha probado los efectos de contagio en los índices de acciones de China, Hong Kong y Taiwán con correlaciones dinámicas en periodos de estabilidad y durante la crisis financiera de Asia incluye a Wang y Nguyen (2013). En un estudio para los índices globales MSCI mensuales de 4 países de América Latina y el índice global de Estados Unidos, Arouri et al. (2013) encuentran fuerte evidencia de que los mercados de acciones de Argentina, Brasil, Chile y México no fueron contagiados durante la crisis mexicana de 1994, la crisis asiática de 1997-1998 y la crisis financiera global de 2008-2009. Otro trabajo que indaga los efectos de contagio de los mercados de acciones de los países PIIGS (países de la Unión Europea integrados por Portugal, Italia, Irlanda, Grecia y España con problemas de déficit y balanza de pagos.), EEUU, Japón y Reino Unido sobre los mercados de acciones de los países BRICS (asociación económica-comercial formada por las cinco economías emergentes más importantes del mundo entre ellos Brasil, Rusia, la India, China y Sudáfrica) incluye a Ahmad et al. (2013). Los resultados señalan que Brasil, China, la India, Rusia y Sudáfrica 
fueron fuertemente afectados por el contagio de la crisis de la zona euro, mientras que los países de Indonesia y Corea del Sur sólo sufrieron interdependencia. Asimismo, Guesmi, Kaabia y Kazi (2013) revelan que las medias de las correlaciones condicionales dinámicas en el periodo estable presentan patrones diferentes a las del periodo de crisis, lo que implica la existencia de efecto contagio en los mercados de acciones de la OCDE, excepto para Alemania, Italia y Reino Unido. A través de un modelo de factor latente, Dungey y Gajurel (2014) proporcionan fuerte evidencia de efectos de contagio procedente de EEUU hacia mercados de acciones desarrollados y emergentes durante la crisis financiera global.

Utilizando modelos de cópulas con parámetros cambiantes en el tiempo, Abbara y Zevallos (2014) estudian el fenómeno de contagio e interdependencia entre los mercados de acciones de EEUU, América Latina (Argentina, Brasil, México), Asia (Japón y Sigapur), Europa (Alemania y Reino Unido) en el periodo del 6 de septiembre al 19 de abril de 2013. Los resultados del análisis bivariado revelan evidencia de contagio entre los mercados de acciones en la crisis asiática-rusa y la crisis subprimefinanciera global. En estudios más recientes, Hemche et al. (2016) analizan la hipótesis de contagio para 10 mercados de acciones desarrollados y emergentes con respecto al mercado de acciones de EEUU en el contexto de la crisis subprime a través de un modelo MGARCH-CCD. Los hallazgos empíricos confirman que la hipótesis de contagio es imposible rechazarla para Francia, Italia, Reino Unido y México al 1\% y para Argentina al 10\%, mientras que para los mercados de acciones de China, Japón, Egipto, Túnez y Marruecos existen vestigios de interdependencia. De la misma manera, Mollaha et al. (2016) encuentran evidencia de contagio en los mercados de acciones desarrollados y emergentes durante la crisis financiera global y la crisis europea, y demuestran que el contagio se propaga de EEUU hacia los mercados de acciones internacionales durante ambos eventos de crisis (en este estudio se utilizaron los índices globales MSCI en dólares estadounidenses de 55 mercados de acciones). Por su parte, Boubaker et al. (2016) proporcionan evidencia de efecto contagio entre el mercado de acciones de EEUU y mercados de acciones desarrollados y emergentes a través de un análisis en los periodos antes y posteriormente a la crisis financiera global, pero utilizando técnicas de cointegración y pruebas de causalidad de Granger.

\section{Datos y Metodología econométrica.}

\subsection{Descripción de los datos.}

Para el estudio de efecto contagio durante la crisis financiera global se utilizan los datos de los precios diarios de cierre del 2 de enero de 2000 al 31 de diciembre de 2009, para los mercados de acciones de 6 países de América Latina y el mercado de acciones de EEUU. El conjunto de datos incluye el Índice de la Bolsa de Valores de Sao Paulo de Brasil (Bovespa), Índice del Mercado de Valores de Buenos Aires (Merval), Índice de Precios Selectivos de Acciones de Chile (IPSA), Índice General de la Bolsa de Valores de Colombia (IGBC), Índice de Precios y Cotizaciones de la Bolsa Mexicana de Valores (IPC), Índice General de la Bolsa de Valores de Lima (IGBVL), Índice S\&P500 de Estados Unidos. La fuente de la información es la base de datos de Bloomberg.

Para la estandarización de las series de los precios, el estudio utiliza el método de interpolación de Lagrange para estimar los datos no disponibles cuando los mercados permanecen cerrados debido a los días festivos u otros eventos especiales. De acuerdo con el horario del meridiano de Greenwich, el sistema de negoción de los diferentes mercados de acciones se encuentra ligeramente no sincronizado: 14:30-21:00 (Nueva York), 14:00-20:00 (Argentina), 14:00-21:00 (Brasil), 13:30-21:00 (Chile), 14:3021:00 (Colombia), 14:30-21:00 (México) y 14:00-21:00 (Perú). Por ello, la serie financiera del S\&P 500 es desfasada un periodo para relajar los problemas de la diferencia de horarios entre el mercado de acciones de Estados Unidos y los 6 mercados de acciones de América Latina. De esta manera, el estudio no considera el uso de la media móvil sobre los rendimientos de dos días para evitar los serios problemas de autocorrelación documentados por Chiang et al. (2007). Para el propósito de estimación, la muestra total se divide en dos muestras que corresponden a los periodos del 2 de enero de 2000 al 30 de 
diciembre de 2005 para la estimación de los parámetros del modelo MGARCH-CCD y 2 de enero de 2006 al 31 de marzo de 2009 para las correlaciones fuera de la muestra.

\subsection{Especificación del modelo econométrico.}

La literatura ha proporcionado evidencia de que la dinámica de las correlaciones de los rendimientos financieros no es constante a través del tiempo, esto es, su tendencia desciende en mercados alcista e incrementa en mercados bajista (Ang \& Bekaert, 1999; Longin \& Solnik, 2001). Asimismo, las correlaciones se incrementan con el grado de integración de los mercados de acciones internacionales (Longin \& Solnik, 1995). Este trabajo emplea el modelo GARCH de correlación condicional dinámico multivariado (MGARCH-CCD) propuesto por Engle (2002), y estima las correlaciones condicionales dinámicas (CCD) entre los mercados de acciones de América Latina y Estados Unidos.

Con respecto a otras alternativas, el modelo MGARCH-CCD tiene la principal ventaja de que permite capturar posibles cambios de régimen en las CCD a través del tiempo, esto es, respuesta de reacción a las malas noticias, innovaciones y crisis financieras. Además, el modelo relaja la carga computacional relacionada con la debilidad de la dimensión cuando el número de variables se incrementan durante la implementación numérica; incluso permite variables explicativas adicionales en la ecuación de la media para explicar el efecto de un factor global. Otra ventaja es que el uso de los residuos estandarizados para la estimación de las CCD resuelve el problema de la heterocedasticidad. De esta manera, el efecto ocasionado por los choques en la volatilidad es mitigado por el procedimiento de estimación del mismo modelo.

El proceso de estimación de las CCD a través del modelo MGARCH-CCD se desarrolla en dos etapas. De esta manera, las ecuaciones de la media condicional para los mercados de acciones son definidas como:

$$
r_{i, t}=\phi_{0}+\phi_{1} r_{i, t-1}+\phi_{2} r_{i, t-1}^{\text {EE.UU. }}+\varepsilon_{t}, \quad \varepsilon_{i, t} \mid \Omega_{t-1} \rightarrow N\left(0, H_{t}\right)
$$

donde $r_{i, t}=\left(r_{1, t}^{\text {Argentina }}, r_{2, t}^{\text {Brasil }}, r_{3, t}^{\text {Chile }}, r_{4, t}^{\text {Colombia }}, r_{5, t}^{\text {Perú }}, r_{6, t}^{\text {EE.UU. }}\right)^{\prime}$ es el vector de rendimientos de los mercados de acciones internacionales en el tiempo $t$. La autocorrelación es corregida a través de un modelo AR(1), es decir, $r_{i, t-1}$. Los rendimientos desfasados un periodo para el mercado de acciones de EE.UU., $r_{i, t-1}^{\mathrm{EE} . U \mathrm{UU}}$ son utilizados para capturar el factor global y las diferencias de horarios de operación en los respectivos mercados. Este último sólo afecta a la media condicional de los mercados de acciones emergentes porque coadyuva a confirmar su impacto sobre los mercados de acciones de América Latina. $H_{t}$ es la matriz de varianzas-covarianzas condicionales, $\varepsilon_{i, t}=\left(\varepsilon_{1, t}^{\text {Argentina }}, \varepsilon_{2, t}^{\text {Brasil }}, \varepsilon_{3, t}^{\text {Chile }}, \varepsilon_{4, t}^{\text {Colombia }}, \varepsilon_{5, t}^{\text {México }}, \varepsilon_{6, t}^{\text {EE.UU. }}\right)^{\prime}$ es el vector del término de error estocástico o ruido blanco. El ajuste de las ecuaciones de la media bajo los modelos $\operatorname{ARMA}(2,2)$ y $\operatorname{AR}(2)$ fueron también considerados en el análisis, pero la estimación por máxima verosimilitud fue más estable a través del modelo AR (1).

En la primera fase se lleva a cabo la estimación de los parámetros de los modelos GARCH estándar, seguido inmediatamente de las CCD en la segunda fase.

$$
H_{t}=D_{t} R_{t} D_{t}
$$

donde $R_{t}$ representa la matriz simétrica de correlaciones dinámicas de dimensión $6 \times 6$, $D_{t}=\operatorname{diag}\left(\sqrt{h_{1, t}}, \sqrt{h_{2, t}}, \sqrt{h_{3, t}}, \sqrt{h_{4, t}}, \sqrt{h_{5, t}}, \sqrt{h_{6, t}}\right)$ es la matriz diagonal de las desviaciones estándar 
condicionales, que se deriva de la estimación de los modelos GARCH estándar para los rendimientos accionarios con $\sqrt{h_{i, t}}$ para $i=1,2,3,4,5,6$.

$$
h_{i, t}=\omega_{i}+\sum_{q=1}^{q_{i}} \alpha_{i q} \varepsilon_{i, t-q}^{2}+\sum_{p=1}^{p_{i}} \beta_{i p} h_{i, t-p}
$$

donde $\omega_{i}$ es una constante, $\alpha_{i}$ y $\beta_{i}$ son los coeficientes ARCH y GARCH, respectivamente.

La matriz de correlaciones condicionales se obtiene de los residuos estandarizados, que son obtenidos de la estimación GARCH. De esta manera, la matriz $R_{t}$ se puede descomponer en:

$$
\begin{aligned}
& R_{t}=Q_{t}^{*-1} Q_{t} Q_{t}^{*-1} \\
& Q_{t}^{*-1}=\operatorname{diag}\left(1 / \sqrt{q_{11, t}}, 1 / \sqrt{q_{22, t}}, 1 / \sqrt{q_{33, t}}, 1 / \sqrt{q_{44, t}}, 1 / \sqrt{q_{55, t}}, 1 / \sqrt{q_{66, t}}\right)
\end{aligned}
$$

donde $Q_{t}=\left|q_{i j, t}\right|$ es una matriz simétrica positiva definida de dimensión $6 \times 6$ de los residuos estandarizados, $\xi_{i, t}=\frac{\varepsilon_{i, t}}{\sqrt{h_{i, t}}}$, que se puede expresar como:

$$
Q_{t}=\left(1-\theta_{1}-\theta_{2}\right) \bar{Q}+\theta_{1} \xi_{i, t-1} \xi_{i, t-1}^{\prime}+\theta_{2} Q_{t-1}
$$

$\bar{Q}=E\left[\xi_{i, t} \xi_{i, t}^{\prime}\right]$ es la matriz de correlaciones no condicionadas de los residuos estandarizados, $\xi_{i, t}$. $\theta_{1}$ y $\theta_{2}$ son parámetros escalares que recogen los efectos de choques previos y las CCD desfasadas sobre las actuales, por lo que deben ser positivos y $\theta_{1}+\theta_{2}<1$.

Las CCD en el tiempo $t$ se pueden expresar de la siguiente manera:

$$
\rho_{i j, t}=\frac{q_{i j, t}}{\sqrt{q_{i i, t} q_{j j, t}}}=\frac{\left(1-\theta_{1}-\theta_{2}\right) q_{i j}+\theta_{1} \xi_{i, t-1} \xi_{i, t-1}^{\prime}+\theta_{2} q_{i j, t-1}}{\left[\left(1-\theta_{1}-\theta_{2}\right) q_{i i}+\theta_{1} \xi_{i, t-1} \xi_{i, t-1}^{\prime}+\theta_{2} q_{i i, t-1}\right]^{1 / 2}\left[\left(1-\theta_{1}-\theta_{2}\right) q_{j j}+\theta_{1} \xi_{i, t-1} \xi_{i, t-1}^{\prime}+\theta_{2} q_{j j, t}\right]^{1 / 2}}
$$

donde $q_{i j}$ es el elemento de la $i$-ésima fila y $j$-ésima columna de la matriz $Q_{t}$.

Finalmente, el proceso de estimación de los parámetros y correlaciones condicionales del modelo MGARCH-CCD se lleva cabo mediante el método de cuasi-máxima verosimilitud. Por lo que, bajo el supuesto de normalidad para las innovaciones de los rendimientos accionarios, la función logarítmica de verosimilitud conjunta se puede expresar de la siguiente manera:

$$
L(\theta)=-\frac{1}{2} \sum_{t=1}^{T}\left[\left(4 \log (2 \pi)+\log \left|D_{t}\right|^{2}+\varepsilon_{t}^{\prime} D_{t}^{-1} \varepsilon_{t}\right)+\left(\log \left|R_{t}\right|+\xi_{t}^{\prime} R_{t}^{-1} \xi_{t}-\xi_{t}^{\prime} \xi_{t}\right)\right]
$$

donde $T$ es el número de observaciones y $\theta$ representa el vector de parámetros a estimar. 


\subsection{Prueba estadística de contagio o interdependencia.}

En esta sección se describe la prueba estadística $t$ que permite probar la hipótesis nula de la ausencia de contagio financiero en los mercados de acciones de Argentina, Brasil, Chile, Colombia, México y Perú durante la crisis financiera global. Sin embargo, la presencia de heterocedasticidad en la volatilidad de mercado puede ocasionar distorsiones en los coeficientes de las correlaciones y proporcionar resultados sesgados para la comprobación del efecto contagio (Forbes \& Rigobon, 2002). Por lo que el objetivo del análisis es comparar las CCD fuera de la muestra en los periodos de estabilidad y crisis financiera a fin de capturar la heterocedasticidad y mejorar la evidencia de contagio o simple interdependencia.

Este estudio utiliza la prueba $t$ para probar la consistencia de las correlaciones fuera de la muestra en los periodos de estabilidad y crisis financiera y, de esta manera, identificar la presencia de simple interdependencia o efecto contagio. Para ello es necesario contrastar las siguientes hipótesis nula y alternativa:

$$
H_{0}: \mu_{\rho}^{\text {crisis }}=\mu_{\rho}^{\text {estable }}, \quad H_{a}=\mu_{\rho}^{\text {crisis }} \neq \mu_{\rho}^{\text {estable }}
$$

donde $\mu_{\rho}^{\text {crisis }}$ y $\mu_{\rho}^{\text {estable }}$ representan las medias poblacionales de las CCD fuera de la muestra durante los periodos de crisis y relativa estabilidad, respectivamente. Asumiendo que el tamaño de las muestras para los diferentes periodos de análisis está definido por $n^{\text {crisis }}$ y $n^{\text {estable }}$, las varianzas poblacionales $\sigma_{\text {crisis }}^{2}$ y $\sigma_{\text {estable }}^{2}$ son diferentes y desconocidas.

Ahora si definimos a las medias de las CCD estimadas fuera de la muestra por el modelo MGARCH-DCC como $\bar{\rho}_{i j}^{\text {crisis }}$ y $\bar{\rho}_{i j}^{\text {estable }}$ con varianzas $s_{\text {crisis }}^{2}$ y $s_{\text {estable }}^{2}$, y bajo la hipótesis nula de simple interdependencia por la ausencia de cambios estructurales en las CCD durante el periodo de crisis o inestabilidad financiera.

El estadístico $t$ se puede expresar de la siguiente manera:

$$
t=\frac{\left(\bar{\rho}_{i j}^{\text {crisis }}-\bar{\rho}_{i j}^{\text {estable }}\right)-\left(\mu_{\rho}^{\text {crisis }}-\mu_{\rho}^{\text {estable }}\right)}{\sqrt{\frac{s_{\text {crisis }}^{2}}{n^{\text {crisis }}}+\frac{s_{\text {estable }}^{2}}{n^{\text {estable }}}}}
$$

Y las varianzas se pueden expresar a través de las siguientes ecuaciones:

$$
S_{\text {crisis }}^{2}=\frac{1}{n^{\text {crisis }}} \sum_{i=1}^{n^{\text {crisis }}}\left(\rho_{i j}^{\text {crisis }}-\bar{\rho}_{i j}^{\text {crisis }}\right)^{2} \text { y } S_{\text {estable }}^{2}=\frac{1}{n^{\text {estable }}} \sum_{i=1}^{n^{\text {cstable }}}\left(\rho_{i j}^{\text {estable }}-\bar{\rho}_{i j}^{\text {estable }}\right)^{2}
$$

El estadístico $t$ sigue una distribución $t$-student bajo la hipótesis nula con $v$ grados de libertad, que se pueden determinar de la siguiente manera:

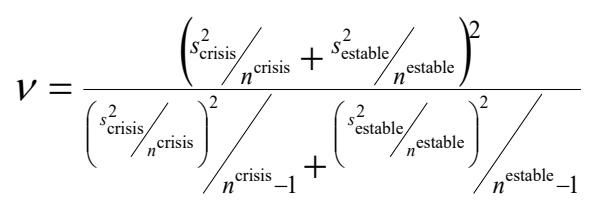

Para obtener resultados robustos del efecto contagio entre los mercados de acciones, el estudio lleva a cabo un análisis bootstrap estacionario para estimar el valor de la probabilidad del estadístico $t$ (para una explicación técnica más detallada del método bootstrap, véase Politis \& Romano (1994)). 
Este procedimiento consiste en la generación de muestras bootstrap $\left(\rho_{b, 1}^{*}, \ldots, \rho_{b, n}^{*}\right)$ con $b=1, \ldots, B$ para las correlaciones fuera de la muestra de los periodos de estabilidad y crisis. Para encontrar la distribución del estadístico bajo la hipótesis nula, el estadístico $t_{b, n}^{*}$ es calculado para cada una de las muestras bootstrap y es comparado con el estadístico $t$. El valor de $B$ debe ser muy grande para obtener resultados confiables y que no afecten a las muestras actuales.

De esta manera, el valor de la probabilidad bootstrap está definido por

$$
\text { valor }-p=B^{-1} \sum_{b=1}^{B} 1_{\left\{t_{b, n}^{*}>t\right\}}
$$

Para el nivel de significación $\alpha$, la hipótesis nula de simple interdependencia se rechaza cuando el valor- $p$ bootstrap es menor a $\alpha$. Para determinar la significancia estadística se generan 10,000 muestras bootstrap a partir de las muestras originales de las CCD estimadas de los diferentes periodos de estabilidad y crisis financiera.

\section{Evidencia empírica.}

\subsection{Análisis de las estadísticas básicas.}

El estudio de los efectos de la crisis financiera global sobre los 6 mercados de acciones de América Latina, se lleva a cabo primero con el análisis de las estadísticas descriptivas antes y durante la crisis. Para ello, las series de los índices son transformados a rendimientos continuos a través de la diferencia entre el $\log a r i t m o$ del precio de cierre actual y el anterior, es decir, $R_{t}=\log P_{t}-\log P_{t-1}$.

En la Tabla 1 se puede observar que la media es positiva para los rendimientos de los 6 mercados de acciones de América Latina y negativa para Estados Unidos en ambos periodos de análisis. Asimismo, la media de los rendimientos tiende a incrementarse en el periodo 2006-2009, excepto para los mercados de acciones de Argentina y Colombia. La desviación estándar presenta valores altos en ambos periodos de análisis, incluso se pueden observar importantes cambios en el periodo 2006-2009, lo que es una evidencia de volatilidad incondicional más pronunciada e inestable para los mercados de acciones, excepto para Argentina. Esto obedece a que en este periodo ocurrieron la crisis subprime y la crisis financiera global. Los resultados del coeficiente sesgo son mixtos en ambos periodos, esto es, positivo para el periodo 2000-2005 y negativo para el periodo 2006-2009 en los mercados de acciones de Argentina, Colombia y Estados Unidos, mientras que lo contrario ocurre para México.

Asimismo, los resultados muestran evidencia de que los rendimientos presentan exceso de curtosis, en particular para el periodo 2006-2009. El supuesto de normalidad es rechazado a un nivel de $5 \%$ para todos los mercados de acciones de acuerdo con la prueba Jarque-Bera. Además, los resultados de la prueba estadística de Ljung-Box, $\mathrm{Q}^{2}(20)$ indican la presencia de heterocedasticidad en los rendimientos de los mercados de acciones en ambos periodos. Este fenómeno común de las series financieras se observa mejor en la Figura 1. 
Tabla 1. Estadísticas básicas de los rendimientos de los mercados de acciones.

\begin{tabular}{lcccccccc}
\hline \multicolumn{1}{c}{ Mercado } & Media & Máximo & Mínimo & Desv. Est. & Sesgo & Curtosis & J-B & $\mathbf{Q}^{2}(\mathbf{2 0})$ \\
\hline Panel A. Periodo $2000-2005$ & & & & & & & \\
Argentina & 0.0707 & 16.1245 & -11.2956 & 2.3520 & 0.2446 & 7.4612 & $1084(0)$ & $300(0)$ \\
Brasil & 0.0327 & 7.3363 & -9.6295 & 1.8771 & -0.2557 & 4.0413 & $72(0)$ & $100(0)$ \\
Chile & 0.0359 & 3.8192 & -3.0915 & 0.8871 & 0.1186 & 4.0776 & $66(0)$ & $238(0)$ \\
Colombia & 0.1038 & 8.8951 & -6.3738 & 1.0841 & 0.6201 & 12.8808 & $5346(0)$ & $135(0)$ \\
México & 0.0463 & 7.0265 & -8.2674 & 1.4238 & -0.0699 & 6.2332 & $564(0)$ & $273(0)$ \\
Perú & 0.0552 & 4.2878 & -5.8872 & 0.8386 & -0.1045 & 7.7890 & $1238(0)$ & $173(0)$ \\
Estados Unidos & -0.0149 & 5.3578 & -6.0057 & 1.2372 & 0.1766 & 4.8130 & $184(0)$ & $553(0)$ \\
Panel A. Periodo $2006-2009$ & & & & & & & \\
Argentina & 0.0414 & 9.7543 & -12.9541 & 1.9136 & -0.8467 & 8.6164 & $1856(0)$ & $1217(0)$ \\
Brasil & 0.0758 & 13.6845 & -12.1883 & 2.0538 & -0.0463 & 8.7175 & $1764(0)$ & $1843(0)$ \\
Chile & 0.0537 & 11.7825 & -6.2154 & 1.1514 & 0.2218 & 14.7456 & $7453(0)$ & $648(0)$ \\
Colombia & 0.0759 & 14.6945 & -11.0521 & 1.6044 & -0.4562 & 14.9713 & $7778(0)$ & $1144(0)$ \\
México & 0.0703 & 10.4486 & -7.2667 & 1.5816 & 0.1583 & 7.6869 & $1187(0)$ & $1017(0)$ \\
Perú & 0.1028 & 12.8245 & -11.4461 & 1.8514 & -0.3578 & 10.2983 & $2821(0)$ & $4249(0)$ \\
Estados Unidos & -0.0064 & 10.9617 & -9.4752 & 1.4842 & -0.2012 & 13.6922 & $6172(0)$ & $2164(0)$ \\
\hline
\end{tabular}

Nota: La tabla reporta las estadísticas básicas de los rendimientos de los mercados de acciones. Los valores entre paréntesis indican las probabilidades de las pruebas de Jarque-Bera y Autocorrelación.

Fuente: Elaboración propia con información de la base de datos de Bloomberg.

Figura 1. Dinámica de los rendimientos de los mercados de acciones de América Latina.

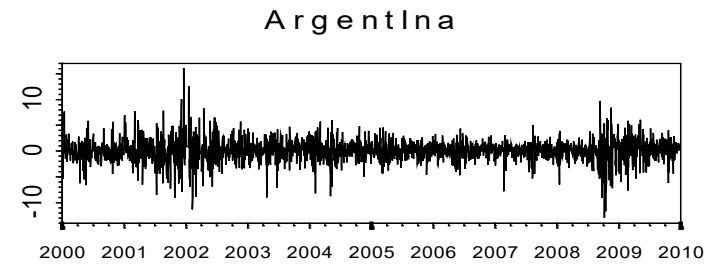

Chile

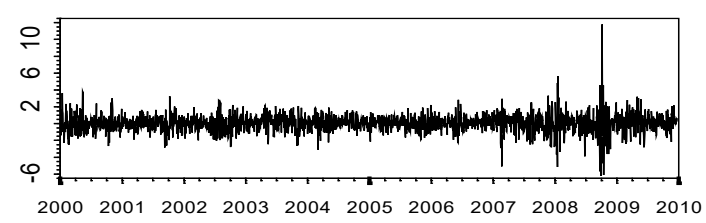

M é xi co

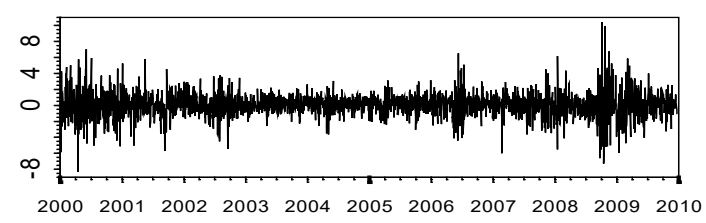

Brasil

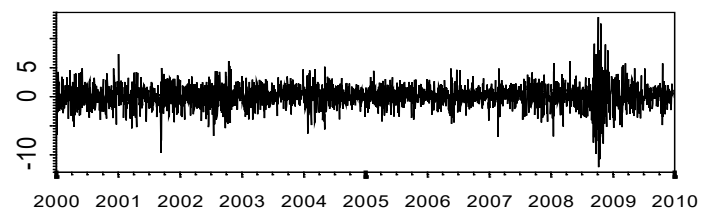

Colombia

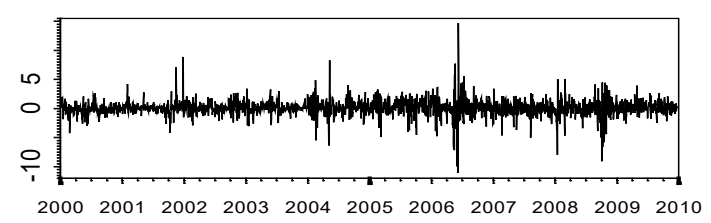

P e rú

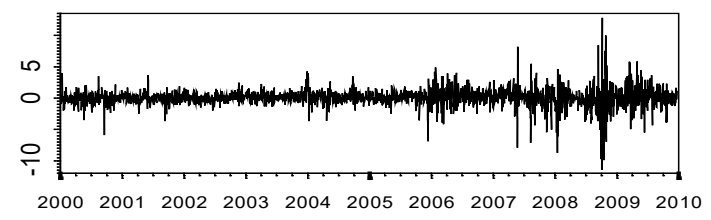

Fuente: Elaboración propia con datos de Bloomberg.

\subsection{Análisis de cointegración.}

El objetivo de esta sección es indagar en las relaciones de cointegración entre los mercados de acciones de Argentina, Brasil, Chile, Colombia, México y Perú con el mercado de acciones de EEUU en los periodos de relativa calma (2000-2005) y crisis (2006-2012), utilizando la aproximación de cointegración de Johansen (1988). Antes de llevar a cabo el análisis de la relación de equilibrio de largo plazo, primero se determina el orden de integración de todas las variables a través de las pruebas de raíz unitaria de Dickey-Fuller aumentada (DFA) y Phillips-Perron (PP). 
Los resultados de las pruebas de raíz unitaria de DFA y PP son mostrados en la Tabla 2. Para los niveles, el valor del estadístico de las pruebas indica que las series son integradas de orden 1, es decir, I(1), lo que implica que todos los índices de los mercados de acciones analizados son no estacionarios en ambos periodos de tranquilidad y crisis. Sin embargo, la presencia de raíces unitarias es rechazada en las primeras diferencias de los índices de los mercados de acciones a un nivel de significancia de $1 \%$, esto es, las series de los rendimientos son estacionarias. En consecuencia, la confirmación de raíces unitarias apoya la presencia de relaciones de cointegración entre el mercado de acciones de EEUU y los 6 mercados de acciones de América Latina considerados.

Tabla 2. Resultados de las pruebas de raíz unitaria.

\begin{tabular}{|c|c|c|c|c|c|c|c|}
\hline & Argentina & Brasil & Chile & Colombia & México & Perú & EEUU \\
\hline \multicolumn{8}{|c|}{ Panel A: Prueba de Raíz Unitaria (2000-2004) } \\
\hline \multicolumn{8}{|l|}{ Niveles } \\
\hline Dicker-Fuller & -1.5425 & -1.0986 & -1.5179 & -1.5837 & -1.4981 & -2.4681 & -1.0845 \\
\hline Phillips-Perron & 0.1772 & -0.5053 & -0.0478 & -0.0166 & -0.1877 & -1.7180 & -1.7421 \\
\hline \multicolumn{8}{|c|}{ Primeras Diferencias } \\
\hline Dicker-Fuller & $-14.7356^{*}$ & $-15.1373 *$ & $-13.9591 *$ & $-13.5204 *$ & $-13.8127^{*}$ & $-13.3221 *$ & $-15.5722 *$ \\
\hline Phillips-Perron & $-31.4201 *$ & $-34.4206^{*}$ & $-32.4118^{*}$ & $-28.9607 *$ & $-29.1632 *$ & $-28.2872 *$ & $-36.3561 *$ \\
\hline \multicolumn{8}{|c|}{ Panel B: Prueba de Raíz Unitaria (2005-2009) } \\
\hline \multicolumn{8}{|l|}{ Niveles } \\
\hline Dicker-Fuller & -1.6173 & -1.7223 & -1.6439 & -2.0471 & -1.1125 & -2.3833 & -1.5262 \\
\hline Phillips-Perron & -1.5026 & -1.2154 & -1.5350 & -1.3135 & -1.6326 & -2.5392 & -1.2077 \\
\hline \multicolumn{8}{|c|}{ Primeras Diferencias } \\
\hline Dicker-Fuller & $-15.4234^{*}$ & $-15.8835^{*}$ & $-14.7824 *$ & $-14.8652 *$ & $-13.4988^{*}$ & $-15.9051^{*}$ & $-15.7213^{*}$ \\
\hline Phillips-Perron & $-31.9756^{*}$ & $-36.5137 *$ & $-34.5164 *$ & $-30.7347^{*}$ & $-29.7973 *$ & $-29.8334 *$ & $-41.0598 *$ \\
\hline
\end{tabular}

Nota: El término * denota significancia a un nivel de 5\%.

Fuente: Elaboración propia con información de la base de datos de Bloomberg.

Asimismo, en la Tabla 3 se reportan los resultados de la prueba de cointegración entre el mercado de acciones de EEUU y cada uno de los mercados de acciones de América Latina analizados. Para evitar estimaciones sesgadas y la presencia de autocorrelación en los residuos, el número de retardos óptimo en la estructura VAR fue determinado de acuerdo al criterio de Hanna-Quinn (HQC). Otras alternativas fueron consideradas para la selección del orden de retardos. Sin embargo, en el análisis se decidió utilizar el HQC sobre los criterios de información de Akaike (AIC) y Schwarz (BIC), debido al hecho de que la tendencia del AIC tiende a sobreestimar el número de retardos y reduce el poder de la prueba. Por su parte, la tendencia del BIC suele subestimar el orden de retardos como consecuencia de la posible pérdida de información importante en la estimación. En términos de confiabilidad, estudios con simulaciones han demostrado que el desempeño del HQC es superior en muestras grandes en comparación a otros criterios (Liev, 2004). Para el periodo de relativa calma, el número de retardos óptimo seleccionado por el HQC equivale a 1 para todos los mercados de acciones. Para el periodo de crisis, el orden de retardos es igual a 3 para todos los mercados de acciones, excepto para Colombia y Perú que equivale a 2 retardos.

De acuerdo con los resultados de las pruebas de la traza y máximo valor propio, la evidencia empírica muestra la existencia de al menos dos vectores de cointegración entre el mercado de acciones de EEUU y los 6 mercados de acciones emergentes de América Latina en los niveles de significación de $1 \%$ y $5 \%$ en ambos periodos de calma y crisis. Los hallazgos obtenidos son consistentes con la evidencia de Boubaker et al. (2016), puesto que la presencia del mismo número de relaciones de cointegración en los dos periodos analizados, indica la existencia de fuertes vínculos entre el mercado de EEUU y los mercados de acciones de Argentina, Brasil, Chile, Colombia, México y Perú. Basado en teorías no relacionadas con crisis, esto significa que los mecanismos de transmisión durante crisis no son significativamente diferentes de los canales de transmisión que ocurren antes de un choque. Por lo que las relaciones de mercado cruzado permanecen sin cambio después de un evento extremo (Forbes 
\& Rigobon, 2001). Este hallazgo confirma que el contagio financiero se propagó a los 6 mercados de acciones de la región de América Latina en la medida que la crisis subprime se fue agudizando.

Tabla 3. Resultados de la prueba de cointegración de Johansen.

\begin{tabular}{|c|c|c|c|c|c|c|c|}
\hline \multirow{2}{*}{\multicolumn{2}{|c|}{ Mercados }} & \multicolumn{2}{|c|}{ Valores Propios } & \multicolumn{2}{|c|}{ Estadístico de la Traza } & \multicolumn{2}{|c|}{ Estadístico del Máximo } \\
\hline & & $2000-2004$ & 2005-2009 & 2000-2004 & 2005-2009 & 2000-2004 & 2005-2009 \\
\hline \multicolumn{8}{|l|}{ Argentina } \\
\hline & $r=0$ & 0.3620 & 0.2405 & $1085^{*}$ & $584 *$ & $580^{*}$ & $355^{*}$ \\
\hline & $r=1$ & 0.3236 & 0.1627 & $504^{*}$ & $229 *$ & $504 *$ & $229 *$ \\
\hline \multicolumn{8}{|l|}{ Brasil } \\
\hline & $r=0$ & 0.3647 & 0.2417 & $1081^{*}$ & $665^{*}$ & $585^{*}$ & $357^{*}$ \\
\hline & $r=1$ & 0.3188 & 0.2126 & $495 *$ & $308 *$ & $495 *$ & $308 *$ \\
\hline \multicolumn{8}{|l|}{ Chile } \\
\hline & $r=0$ & 0.3688 & 0.2193 & $1015^{*}$ & $565^{*}$ & $594 *$ & $319 *$ \\
\hline & $r=1$ & 0.2787 & 0.1735 & $421 *$ & $245^{*}$ & $421 *$ & $245^{*}$ \\
\hline \multicolumn{8}{|l|}{ Colombia } \\
\hline & $r=0$ & 0.3642 & 0.2743 & $968 *$ & $708 *$ & $584^{*}$ & $414^{*}$ \\
\hline & $r=1$ & 0.2576 & 0.2039 & $384^{*}$ & $294 *$ & $384 *$ & $294 *$ \\
\hline \multicolumn{8}{|l|}{ México } \\
\hline & $r=0$ & 0.3622 & 0.2293 & $1098^{*}$ & $645^{*}$ & $580^{*}$ & $335^{*}$ \\
\hline & $r=1$ & 0.3303 & 0.2132 & $517^{*}$ & $309 *$ & $517^{*}$ & $309 *$ \\
\hline \multicolumn{8}{|l|}{ Perú } \\
\hline & $r=0$ & 0.3629 & 0.2790 & $955^{*}$ & $789 *$ & $582^{*}$ & $422 *$ \\
\hline & $r=1$ & 0.2511 & 0.2474 & $373 *$ & $367^{*}$ & $373^{*}$ & $367 *$ \\
\hline
\end{tabular}

Nota: los valores críticos se tomaron de Osterwald-Lenum (1992). El término * indica que la hipótesis nula es rechazada a un nivel de significancia de $5 \%$.

Fuente: Elaboración propia con información de la base de datos de Bloomberg.

\subsection{Estimación de los parámetros.}

En la Tabla 4 se muestran los resultados dentro de la muestra del modelo MGARCH-CCD entre el mercado de acciones de EEUU y los mercados de acciones de América Latina para el periodo 20002006, así como las pruebas de diagnóstico. El término constante de la ecuación de la media condicional, $\phi_{0}$ es estadísticamente significativo en los niveles de $1 \%$ y $5 \%$ para todos los índices bursátiles. El coeficiente del modelo autorregresivo de primer orden, $\phi_{1}$ es positivo y significativo en los niveles convencionales para los mercados de acciones de América Latina, excepto Brasil. En el caso de EEUU, el coeficiente es negativo y significativo al nivel de 1\%. Los hallazgos indican la presencia de un efecto de retroalimentación en el mercado de acciones de EEUU, mientras que en los mercados de acciones de América Latina existe fuerte evidencia de persistencia en los rendimientos financieros por las fricciones de precios o ajustes parciales. Los rendimientos de EEUU tienen un efecto desfasado positivo significativo sobre los rendimientos de acciones de Chile, Colombia, México y Perú, lo que confirma el papel dominante del mercado de acciones desarrollado como factor de perturbación internacional sobre los mercados de acciones de la región de América Latina. Estos hallazgos se encuentran en línea con los resultados del grueso de la literatura sobre integración financiera con el mercado de EEUU.

De acuerdo con la estimación de la ecuación de la varianza, los resultados revelan que los coeficientes de los términos ARCH y GARCH son altamente significativos y con valores positivos para todos los índices de acciones, lo que justifica lo apropiado de la especificación GARCH $(1,1)$ y confirma que la dinámica de la volatilidad cambia con el tiempo. Además, los valores de los coeficientes GARCH son más grandes que los coeficientes $\mathrm{ARCH}$, lo que indica que los mercados de acciones de América Latina pueden ajustarse por sí mismos a las condiciones de mercado. Esto significa que los choques exógenos tienen un impacto más pequeño en la volatilidad condicional que el alcanzado por el ajuste automático (para una explicación más detallada, véase Huang et al. (2000)). Otro hallazgo importante 
es que la suma de los coeficientes ARCH y GARCH es menor a 1. Este resultado señala la presencia de un alto grado de persistencia en la volatilidad de los rendimientos de los 7 mercados de acciones, lo que conlleva a satisfacer la condición de convergencia y varianza positiva en el proceso GARCH.

Tabla 4. Resultados del modelo MGARCH-CCD para el periodo 2000-2006.

\begin{tabular}{|c|c|c|c|c|c|c|c|c|c|}
\hline & \multicolumn{3}{|c|}{ Ecuación de la media condicional } & \multicolumn{3}{|c|}{ Ecuación de la varianza condicional } & \multirow[b]{2}{*}{$\alpha+\beta$} & \multirow[b]{2}{*}{$Q(p)$} & \multirow[b]{2}{*}{$Q^{2}(p)$} \\
\hline & $\phi_{0}$ & $\phi_{1}$ & $\phi_{2}$ & $\omega$ & $\alpha$ & $\beta$ & & & \\
\hline \multirow[t]{2}{*}{ Estados Unidos } & $0.0360 * *$ & & $-0.0529 *$ & $0.0090^{*}$ & $0.0653^{*}$ & $0.9287^{*}$ & 0.9940 & 19.2302 & 12.8027 \\
\hline & $(0.0164)$ & & $(0.0193)$ & $(0.0036)$ & $(0.0080)$ & $(0.0085)$ & & {$[0.2252]$} & {$[0.6352]$} \\
\hline \multirow[t]{2}{*}{ Argentina } & $0.1058^{* *}$ & $0.0658^{* *}$ & -0.0013 & $0.0734^{*}$ & $0.0813^{*}$ & $0.9048 *$ & 0.9861 & 27.0453 & 16.6575 \\
\hline & $(0.0442)$ & $(0.2646)$ & $(0.0445)$ & $(0.0234)$ & $(0.0112)$ & $(0.0124)$ & & [0.1024] & {$[0.4213]$} \\
\hline \multirow{2}{*}{ Brasil } & $0.1169^{*}$ & 0.0217 & 0.0043 & $0.1030 * *$ & $0.0532 *$ & $0.9147^{*}$ & 0.9679 & 22.9743 & 13.7831 \\
\hline & $(0.0414)$ & $(0.0254)$ & $(0.0454)$ & $(0.0412)$ & $(0.0119)$ & $(0.0216)$ & & [0.1203] & {$[0.3443]$} \\
\hline \multirow[t]{2}{*}{ Chile } & $0.0578^{*}$ & $0.2008^{*}$ & $0.0364 * *$ & $0.0302^{*}$ & $0.0931^{*}$ & $0.8619 *$ & 0.9550 & 28.3212 & 11.6312 \\
\hline & $(0.0182)$ & $(0.0247)$ & $(0.0185)$ & $(0.0087)$ & $(0.0161)$ & $(0.0238)$ & & [0.1612] & {$[0.2983]$} \\
\hline \multirow[t]{2}{*}{ Colombia } & $0.0714^{*}$ & $0.2241 *$ & $0.0609 *$ & $0.1481^{*}$ & $0.2141^{*}$ & $0.6979 *$ & 0.9120 & 25.1235 & 18.8630 \\
\hline & $(0.0186)$ & $(0.0209)$ & $(0.0142)$ & $(0.0236)$ & $(0.0230)$ & $(0.0313)$ & & [0.1376] & {$[0.2564]$} \\
\hline \multirow[t]{2}{*}{ México } & $0.1098^{*}$ & $0.0733^{*}$ & $0.0532 * * *$ & $0.0182^{*}$ & $0.0481 *$ & $0.9400^{*}$ & 0.9881 & 20.2365 & 9.2314 \\
\hline & $(0.0268)$ & $(0.0251)$ & $(0.0311)$ & $(0.0059)$ & $(0.0087)$ & $(0.0105)$ & & [0.1387] & {$[0.3235]$} \\
\hline \multirow[t]{2}{*}{ Perú } & $0.0633^{*}$ & $0.1934 *$ & $0.0940 *$ & $0.0805^{*}$ & $0.1931 *$ & $0.7090^{*}$ & 0.9021 & 27.2134 & 22.2153 \\
\hline & $(0.0184)$ & $(0.0274)$ & $(0.0159)$ & $(0.0186)$ & $(0.0304)$ & $(0.0181)$ & & [0.0923] & {$[0.1956]$} \\
\hline \multicolumn{10}{|c|}{ Ecuación de las correlaciones condicionales dinámicas multivariadas } \\
\hline$\theta_{1}$ & $0.0233^{*}$ & $(0.0099)$ & & & & & & & \\
\hline$\theta_{2}$ & $0.7198^{*}$ & $(0.0197)$ & & & & & & & \\
\hline
\end{tabular}

Fuente: Elaboración propia con información de la base de datos de Bloomberg.

Los últimos dos renglones muestran las estimaciones de los parámetros $\theta_{1}$ y $\theta_{2}$ de las CCD de la ecuación (7). Ambos parámetros estimados son estadísticamente significativos al 1\%, lo que implica co-movimientos cambiantes en el tiempo entre los mercados de acciones de América Latina y el mercado de acciones de EEUU. Además, la magnitud del parámetro $\theta_{2}$ confirma que las CCD muestran un alto grado de persistencia. Finalmente, los términos $Q(20)$ y $Q^{2}(20)$ representan los estadísticos de las pruebas de Ljung-Box para los residuales estandarizados simples y cuadrados. Los resultados indican que no existe evidencia significativa de correlación serial y heterocedasticidad en los residuales estandarizados simples y cuadrados hasta términos de orden 20. Estos resultados confirman que la especificación del modelo MGARCH-CCD es apropiada para capturar las características comunes de los rendimientos de los mercados de acciones, en particular de las economías emergentes.

\subsection{Dinámica de las correlaciones condicionales dinámicas.}

En esta sección se analiza la evolución de las CCD fuera de la nuestra entre el mercado de acciones de EEUU y los 6 mercados de acciones de América Latina durante el periodo 2006-2009. El tamaño de la muestra total equivale a 776 observaciones, que se divide en dos submuestras.

La Figura 2 describe el comportamiento dinámico de los coeficientes de correlación entre el mercado de acciones de EEUU y los 6 mercados de acciones de América Latina en dos periodos. El primero corresponde al periodo estable que comprende del 3 de abril 2006 al 29 de septiembre de 2007 y el segundo al periodo de crisis financiera del 1 de octubre de 2007 al 31 de marzo de 2009. Los periodos de estabilidad y crisis fueron seleccionados de acuerdo a los eventos que agudizaron la crisis subprime y crisis financiera global. Algunas fechas claves son el mes de junio de 2007 cuando los primeros productos estructurados con hipotecas subprime o basura comienzan a derrumbarse en el banco Bear Stearns, lo que generó escasez de crédito y desconfianza en bancos como Merrill Lynch, 
JPMorgan Chase, Citigroup y Goldman Sachs el 17 de septiembre del mismo año. Estos problemas propiciaron el inicio de la crisis financiera con la absorción del banco Bear Stearns, y que se agravó con la caída de Lehman Brothers y la compra de Merrill Lynch por parte de Bank of America el 17 de septiembre de 2008.

La Figura 2 revela que los 6 mercados de acciones de América Latina presentan un alto grado de correlación con respecto al mercado de EEUU, excepto para los países de Colombia y Chile. En el análisis de la estructura de las CCD se puede observar un comportamiento similar al de un proceso no estacionario para cada par de series a través del tiempo; es decir, regímenes de volatilidad baja y alta para las CCD, ocasionado por el alto grado de persistencia. De ahí, la importancia de tomar en cuenta el factor global alimentado por el efecto de los rendimientos del mercado de acciones de EEUU hacia los rendimientos de los mercados de acciones emergentes. La tendencia de las CCD se mantiene oscilando ligeramente durante el periodo del 3 de abril de 2006 al 27 de febrero de 2007, en particular para los mercados de acciones de Chile y Brasil. Sin embargo, las CCD comienzan a experimentar alta volatilidad a partir del 28 de febrero del mismo año, al incrementarse $99.76 \%$ para Perú, $89.38 \%$ para Argentina y $61.94 \%$ para Colombia. Este incremento en las CCD aunque breve, particularmente se deriva después del anuncio de banco HSBC, uno de los principales participantes en el sector inmobiliario de EEUU, de que mayores pérdidas se esperaban en las carteras de deuda hipotecaria.

Figura 2. Comportamiento de las correlaciones condicionales dinámicas en 2006-2009.
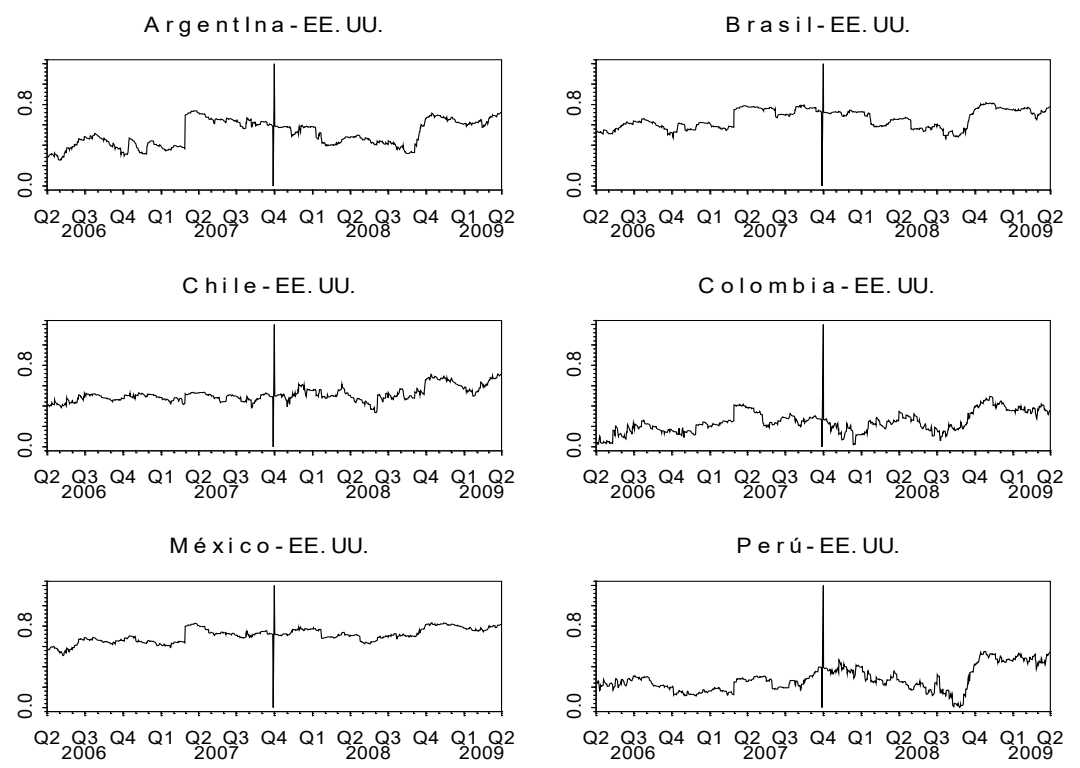

Fuente: Elaboración propia con información de la base de datos de Bloomberg.

Sin embargo, esta tendencia alcista fue interrumpida rápidamente cuando las CCD experimentaron una caída dramática y prolongada durante el periodo del 17 de abril de 2007 y 4 de septiembre de 2008, en particular Perú, Colombia, Argentina y Brasil cuando su grado de correlación disminuyó de 0.3006 a $0.0326,0.3494$ a $0.1741,0.6977$ a 0.3273 y 0.7805 a 0.5202 , respectivamente. Otro importante hallazgo es que durante este mismo periodo los mercados de acciones de México y Chile se han mantenido altamente correlacionados, a pesar de que sus correlaciones disminuyeron ligeramente de 0.7748 a 0.7002 y 0.5291 a 0.4795 en el mismo orden. No obstante, el efecto contagio es más visible durante la agravación de la crisis financiera global que durante la crisis subprime. Esto obedece a que el efecto en las CCD fue más drástico y prolongado, que inicia el 24 de septiembre de 2008 y termina hasta el 31 de marzo de 2009, lo que reduce la inversión de portafolio con instrumentos financieros tanto de los mercados de EEUU como de los mercados de acciones emergentes debido a que se reducen los beneficios de la diversificación. De esta manera, los hallazgos indican que las CCD cambian a través del tiempo, pero con diferente magnitud en cada mercado de acciones. Más 
específicamente, las CCD son más altas para los mercados de acciones de Argentina, Brasil, Chile y México, pero menos volátiles que en los mercados de acciones de Colombia y Perú.

\subsection{Resultados de la prueba de contagio.}

En esta sección se prueba la existencia de contagio financiero a través del estadístico $t$. Esta prueba estadística rechaza o acepta la hipótesis nula de que los coeficientes de las CCD no presentan un comportamiento diferente en periodos de estabilidad y crisis financieras. En este estudio se replican las CCD para ambos periodos de estabilidad y crisis usando 10,000 replicaciones bootstrap estacionarias para determinar el valor- $p$ bootstrap.

En la Tabla 5 se muestran el promedio y varianza de las correlaciones estimadas fuera de la muestra, el estadístico $t$ promedio de las 10,000 réplicas bootstrap y el valor- $p$ de la prueba de efecto contagio financiero entre los 6 mercados de acciones de América Latina y el mercado de EEUU. Los resultados muestran que las CCD son significativamente positivas y evidentemente más altas durante el periodo de crisis en comparación a las del periodo estable. De acuerdo con el incremento en términos porcentuales, los mercados de acciones de Perú, Colombia, Chile y México parecen ser los más influenciados por los efectos de contagio de la crisis financiera global, al registrar cambios del $45.41 \%$, $22.72 \%, 13.26 \%$ y $9.72 \%$ respectivamente. Este hallazgo es alimentado por el incremento de la varianza en el periodo de crisis, aunque en el caso de México la varianza no se mueve en la misma dirección del coeficiente de correlación promedio. Este hecho puede ser justificado por la fuerte relación económica y comercial sostenida entre ambos países desde 1994.

Tabla 5. Coeficientes de correlaciones dinámicas y prueba de contagio financiero.

\begin{tabular}{llccccc}
\hline & \multicolumn{5}{c}{ Correlación } & \\
\cline { 2 - 5 } & \multicolumn{2}{c}{ Media } & \multicolumn{2}{c}{ Varianza } & Estadístico- $t$ & \\
\hline Mercado & Estable & Crisis & Estable & Crisis & $H_{0}: \mu_{\rho}^{\text {crisis }}=\mu_{\rho}^{\text {estable }}$ & Valor- $p$ \\
\hline Argentina & $0.4913^{*}$ & $0.5307^{*}$ & 0.0195 & 0.0119 & 4.3697 & 0.5177 \\
Brasil & $0.6481^{*}$ & $0.6700^{*}$ & 0.0082 & 0.0083 & $8.3574^{*}$ & 0.0231 \\
Chile & $0.4797^{*}$ & $0.5433^{*}$ & 0.0013 & 0.0066 & $14.0704^{*}$ & 0.0341 \\
Colombia & $0.2174^{*}$ & $0.2668^{*}$ & 0.0080 & 0.0115 & $6.9586^{*}$ & 0.0185 \\
México & $0.6747^{*}$ & $0.7403^{*}$ & 0.0045 & 0.0026 & $14.7405^{*}$ & 0.0289 \\
Perú & $0.2277^{*}$ & $0.3311^{*}$ & 0.0039 & 0.0194 & $13.3443^{*}$ & 0.0142 \\
\hline
\end{tabular}

Fuente: Elaboración propia con información de la base de datos de Bloomberg.

Los resultados del estadístico $t$ comprueban si las CCD entre cualquier mercado de acciones de América Latina y el mercado de EEUU son consistentes en periodos de crisis y estabilidad. Cuando las CCD son significativas y la hipótesis nula es rechazada por el valor- $p$ bootstrap, el cual debe ser menor al nivel de significación de 5\%; este hecho evidencia la existencia de contagio financiero. Sin embargo, cuando las CCD son significativas y la hipótesis nula no es rechazada por el alto valor- $p$ bootstrap alcanzado, implica la existencia de una relación de interdependencia. En este sentido, los resultados del valor- $p$ bootstrap revelan que las CCD estimadas fuera de la muestra presentan un comportamiento significativamente diferente en los periodos de estabilidad y crisis financiera, lo que implica fuerte evidencia de la existencia de contagio financiero de la crisis financiera global sobre la mayoría de los mercados de acciones. Por lo que la dependencia de los mercados de acciones de América Latina tiende a incrementarse durante los periodos de turbulencia financiera y, como consecuencia, los coeficientes de correlación son más grandes con respecto al valor promedio.

Por otra parte, los hallazgos son relevantes para los reguladores porque les proporciona información para crear sólidos mecanismos que permitan controlar el tipo de cambio ante la posibilidad de fugas de capital de los mercados de acciones de América Latina hacia otros mercados de acciones del mundo, principalmente durante periodos de crisis financieras o turbulencia financiera. Asimismo, estos resultados contradicen los hallazgos empíricos del trabajo de Arouri et al. (2013), quienes no 
apoyan estadísticamente la evidencia de contagio financiero entre el mercado de acciones de EEUU y los mercados de acciones de Argentina, Brasil, Chile y México. Aunque el efecto de contagio financiero para Argentina desaparece durante la crisis financiera global, este hallazgo significa la fuerte presencia del fenómeno de interdependencia, y que los inversionistas institucionales y administradores de portafolios pudieron haber obtenido beneficios de la diversificación internacional debido a la inclusión de títulos de capital de estos mercados de acciones en sus portafolios de inversión.

\subsection{Relación entre la volatilidad condicional y correlaciones condicionales.}

En esta sección se lleva a cabo un análisis del impacto de la volatilidad condicional para explicar las características cambiantes en el tiempo de las correlaciones condicionales estimadas fuera de la muestra en periodos de crisis y estabilidad. En este tema, la literatura ha proporcionado evidencia de que la estructura de dependencia de los mercados de acciones internacionales tiende a comportarse más inestable cuando el grado de riesgo es más alto (Connoly, Strivers \& Sun, 2007; Aydemir, 2008; Ca, Yeutien \& Li, 2009).

Para ello, el análisis de la regresión por cuantiles es utilizado, aunque este ejercicio se puede realizar con una simple regresión lineal. El modelo de regresión por mínimos cuadrados es una técnica efectiva porque la variable dependiente es definida como una función lineal de una o varias variables independientes, que está sujeta a un término de error aleatorio y su estimador depende de la relación promedio entre la variable dependiente y las variables independientes para ciertos niveles específicos. Sin embargo, la técnica comienza a perder efectividad cuando se intenta ir más allá del valor medio o hacia las observaciones extremas a fin de estudiar las relaciones a través de las colas inferior y superior de la distribución. De acuerdo con el trabajo de Koenker y Bassett (1978), la regresión por cuantiles estima la función cuantil condicional de la variable dependiente con base en las variables independientes para diferentes cuantiles de la distribución de la variable dependiente.

En términos generales, el modelo de regresión por cuantiles para las correlaciones entre los mercados de acciones se puede expresar de la siguiente manera:

$$
Q_{\rho_{t}}\left(\tau \mid \Omega_{t}\right)=\alpha(\tau)+\beta(\tau) h_{i, t}^{1 / 2}+\gamma(\tau) h_{j, t}^{1 / 2}
$$

donde $\rho_{t}$ es la variable dependiente que corresponde a las correlaciones entre los mercados de acciones de EEUU y América Latina, es decir, $\rho_{i j, t}$ tal que $i=$ Estados Unidos y $j=$ Argentina, Brasil, Chile, Colombia, México y Perú, $\Omega_{t}$ es el conjunto de información disponible en el tiempo $t, h_{i, t}^{1 / 2}$ representa la volatilidad condicional del mercado de acciones de EEUU y $h_{j, t}^{1 / 2}$ la volatilidad condicional para cada uno de los mercados de acciones de la región de América Latina. Por su parte, $\alpha(\tau), \beta(\tau)$ y $\gamma(\tau)$ son los parámetros a estimar en los diferentes cuantiles de la distribución. Para un análisis más profundo de los estimadores $\beta(\tau)$ y $\gamma(\tau)$, valores positivos y significativos indican que los coeficientes de correlación condicional sufren incrementos con la dinámica de la volatilidad de los mercados de acciones de EEUU y los países emergentes latinoamericanos, mientras que los coeficientes negativos y significativos provocan un efecto negativo en las correlaciones condicionales.

Para un cuantil fijo $\tau$, el vector de parámetros $[\alpha(\tau), \beta(\tau), \gamma(\tau)]$ se puede estimar al minimizar la suma ponderada de los errores absolutos de la siguiente manera:

$$
\left.[\alpha(\tau), \beta(\tau), \gamma(\tau)]=\underset{\alpha(\tau), \beta(\tau), \gamma(\tau)}{\arg \min } E \mid \phi_{\tau}\left(\rho_{t}-\alpha(\tau)-\beta(\tau) h_{t, i}-\gamma(\tau) h_{t, j}\right)\right]
$$


donde $\phi_{\tau}(z)$ es definida como la función de pérdidas, que establece diferentes pesos sobre los residuos positivos y negativos. Los métodos de programación lineal son aplicados para obtener soluciones óptimas del problema de minimización. En el caso de soluciones interiores bajo algunas condiciones de regularidad, el cuantil con base en el estimador del vector de parámetros $[\alpha(\tau), \beta(\tau), \gamma(\tau)]$ es asintóticamente normal. Sin embargo, la estimación directa de la matriz de varianza-covarianza asintótica no siempre cumple ciertas características satisfactorias. En consecuencia, el método bootstrap es sugerido en la literatura para la inferencia estadística de los parámetros de la regresión por cuantiles.

En la Tabla 6 se reportan los resultados de la regresión por cuantiles para cada una de las variables explicativas. Para obtener un análisis robusto se utilizaron 13 cuantiles: $0.01,0.05,0.10,0.25,0.40$, $0.50,0.60,0.75,0.90,0.95,0.975,0.99,0.999$, así como 5000 replicaciones bootstrap para cada una de las estimaciones. La mayoría de los parámetros $\alpha(\tau)$ son positivos y significativos excepto en el mercado de acciones de Perú para el cuantil 0.01. En el caso de los parámetros $\beta(\tau)$ y $\gamma(\tau)$ se pueden observar resultados mixtos, por ejemplo, para los mercados de acciones de Chile, Colombia y Perú existe evidencia de un efecto significativo de la volatilidad del mercado de acciones de EEUU en el incremento de las correlaciones condicionales durante el periodo de análisis en todos los cuantiles, asociados con coeficientes $R^{2}$ relativamente altos entre 0.1165 y 0.5554 . En tanto que el efecto de la volatilidad de los mercados de América Latina es significativamente positivo para las correlaciones condicionales entre EEUU y Chile para los cuantiles del 0.05 al 0.75 y 0.99 al 0.999 y mixto para los casos de Colombia y Perú, aunque débil debido al hecho de que el parámetro estimado carece de significancia en 5 de los 13 cuantiles.

Para el caso de Argentina, las correlaciones condicionales se incrementan significativamente con la volatilidad del mercado de acciones de EEUU en los cuantiles del 0.01 al 0.60 y disminuyen en los cuantiles del 0.90 al 0.999 con un poder explicativo de regresión entre $0.1655-0.2538$ y 0.0569-0.0763, respectivamente. Asimismo, la relación entre las correlaciones condicionales y la volatilidad del mercado de acciones de Argentina se mantiene significativamente positiva para cuantiles entre 0.750.999 , aunque con coeficientes $R^{2}$ relativamente pequeños, es decir, entre 0.0569 y 0.0763 . Para Brasil, las correlaciones condicionales exhiben una relación positiva significativa con la volatilidad del mercado de acciones de EEUU para los cuantiles entre 0.10-0.75 y una relación negativa significativa para los cuantiles entre $0.90-0.975$ con coeficientes $R^{2}$ que oscilan entre 0.0839 y 0.2322 . Mientras que la relación entre las correlaciones condicionales y volatilidad del mercado de acciones de Brasil es significativamente positiva, excepto en los cuantiles del 0.40 al 0.75 .

En el caso de México, los resultados revelan una relación positiva y significativa con la volatilidad del mercado de acciones de EEUU para cuantiles menores o iguales a 0.90 con un poder explicativo de regresión entre 0.2108 y 0.3361 , mientras que en los cuantiles mayores o iguales a 0.95 el efecto es no significativo. En contraste, las correlaciones condicionales tienden a disminuir significativamente con la volatilidad del mercado de acciones de México para los cuantiles 0.40 y 0.50 , seguido por una relación positiva en los cuantiles del 0.75 al 0.975 . De acuerdo con la magnitud y significancia de los parámetros $\beta(\tau)$ y $\gamma(\tau)$, la intensidad de la volatilidad del mercado de acciones de EEUU tiene mayor impacto positivo en la dinámica de las correlaciones condicionales que el efecto de la volatilidad de los mercados de acciones emergentes con la excepción de Argentina y Brasil, particularmente cuando la volatilidad del mercado de acciones desarrollado se encuentra por arriba del promedio. Estos hallazgos proporcionan fuerte evidencia de que el contagio financiero fue transmitido del mercado de acciones de EEUU hacia los mercados de acciones de América Latina durante la crisis financiera global. 
Tabla 6. Estimación de la regresión por cuantiles.

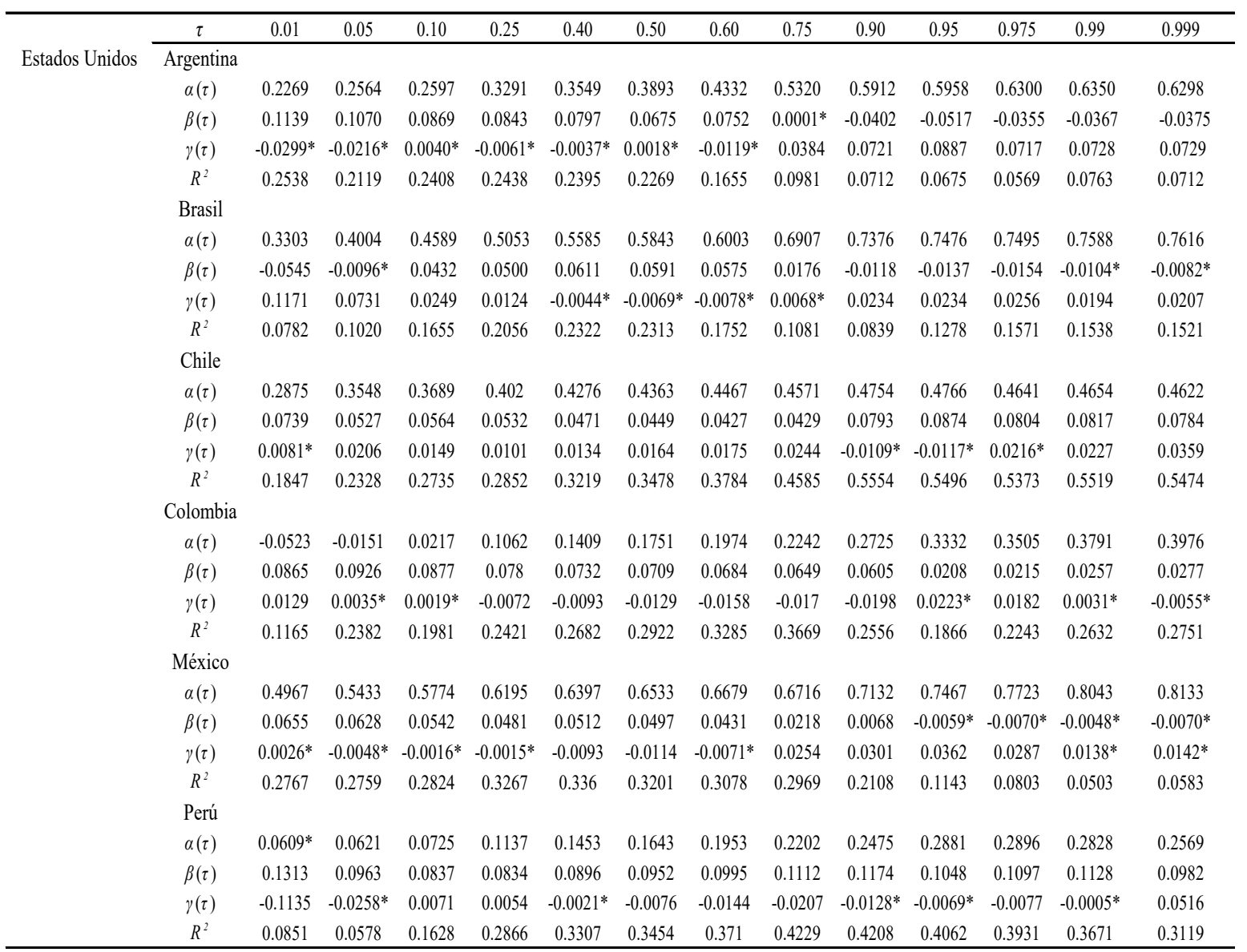

Nota: El término * indica no significancia al nivel de $5 \%$.

Fuente: Elaboración propia con información de la base de datos de Bloomberg.

\section{Conclusiones.}

Este trabajo analiza el comportamiento de las correlaciones condicionales dinámicas en diferentes periodos de estabilidad y crisis financiera para probar la existencia de efecto contagio entre el mercado de acciones de EEUU y los mercados de acciones de Argentina, Brasil, Chile, Colombia, México y Perú. Los resultados revelan que las correlaciones condicionales dinámicas tienden a incrementarse notablemente en el periodo de crisis, pero con diferente magnitud para cada mercado de acciones. Este hallazgo proporciona nueva evidencia de que los mercados de acciones de Brasil, Chile, Colombia, México y Perú fueron más vulnerables al contagio financiero como consecuencia del comportamiento de los inversionistas institucionales en periodos de alta volatilidad. Por lo que la presencia de contagio financiero en la región ha limitado las oportunidades de la diversificación internacional y la obtención de rendimientos superiores para los inversionistas institucionales en el corto plazo. Esto se atribuye a la mayor presencia de riesgo sistemático en los portafolios formados por activos financieros de estos mercados de acciones durante la crisis financiera global. Aunque la evidencia de interdependencia aún existe en la región entre los mercados de acciones de EEUU y Argentina. Los inversionistas deben considerar las condiciones y características de los mercados bursátiles al momento de invertir en títulos de capital.

Los hallazgos del estudio tienen importantes implicaciones económicas y financieras para las autoridades gubernamentales, inversionistas y administradores de riesgos. En este sentido, los diseñadores de la política monetaria deben aprovechar la información generada para instrumentar apropiados mecanismos que garanticen la liquidez en los mercados de acciones emergentes y que 
coadyuven a inmunizar el impacto de los choques externos en sistemas financieros frágiles. Los inversionistas contarán con mejor conocimiento del riesgo sistémico y beneficios de la diversificación para la eficiente asignación de capital en mercados altamente volátiles. Los hallazgos proporcionan información relevante a los administradores de riesgo para el diseño de óptimas estrategias de cobertura que contribuyan a reducir los efectos negativos del contagio financiero en las inversiones de portafolio. Finalmente, el análisis deja abierta una agenda de trabajo para capturar los diferentes niveles de asimetría en el modelo MGARCH-CCD y mejorar la evidencia del contagio financiero.

\section{Referencias}

Abbara, O., \& Zevallos, M. (2014). Assessing stock market dependence and contagion. Quantitative Finance, 14 (9), 1627-1647.

Ahmad, W., Sehgal, S., \& Bhanumurthy, N.R. (2013). Eurozone crisis and BRIICKS stock markets: Contagion or market interdependence? Economic Modelling, 33, 209-225.

Ang, A., \& Bekaert, G. (1999). International asset allocation with time-varying correlations. NBERWorking Paper No. 7056.

Arouri, M. E., Lahiani, A., \& Nguyen, D. (2013). Equity market comovements and financial contagion: a study Latin America y United States. Bankers, Markets \& Investors, 125 (SeptemberOctober), 17-29.

Aydemir, A.C. (2008). Risk sharing and counter-cyclical variation in market correlations. Journal of Economic Dynamics and Control, 32(10), 3084-3112.

Baur, D.G. (2003). What is co-movement? EUR Working Paper No. 20759 EN.

Bouaziz, M.C., Selmi, N., \& Boujelbene, Y. (2012). Contagion effect of the Subprime financial crisis: Evidence of DCC multivariate GARCH models. European Journal of Economics, Finance and Administrative Sciences, 44(1), 66-76.

Boubaker, S., Jouinic, J., \& Lahianid, A. (2016). Financial contagion between the US and selected developedand emerging countries: The case of the subprime crisis. The Quarterly Review of Economics and Finance, 61(August), 14-28.

Cai, Y., Yeutien, R., \& Li, D. (2009). Explaining international stock correlations with CPI fluctuations and market volatility. Journal of Banking and Finance, 33(11), 2026-2035.

Celik, S. (2012). The more contagion effect on emerging markets: The evidence of DCC-GARCH model. Economic Modelling, 29(5), 1946-1959.

Chiang, T.C., Jeon, B.N., \& Li, H. (2007). Dynamic correlation analysis of financial contagion: Evidence from Asian markets. Journal of International Money and Finance, 26(7) 1206-1228.

Cho, J.H., \& Parhizgari, A.M. (2008). East Asian financial contagion under DCC-GARCH. The International Journal of Banking and Finance, 6(1), 17-30.

Connolly, R.A., Strivers, C., \& Sun, L. (2007). Commonality in the time-variation of stock-stock and stock-bond return comovements. Journal of Financial Markets, 10(2), 192-218.

Corsetti, G., Pericoli, M., \& Sbracia, M. (2005). Some contagion, some Interdependence: More pitfalls in tests of financial contagion. Journal of International Money and Finance, 24(8), 1177-1199. 
Dungey, M., \& Gajurel, D. (2014). Equity market contagion during the global financial crisis: Evidence from the world's eighth largest economies. Economic Systems, 38(2), 161-177.

Engle, R.F. (2002). Dynamic conditional correlation: a simple class of multivariate generalized autoregressive conditional heteroskedasticity models. Journal of Business and Economics Statistics, 20(3), 339-350.

Forbes, K., \& Rigobon, R. (2001). Contagion in Latin America: Definitions, measurement, and policy implications. Economia, 1(2), 1-46.

Forbes, K., \& Rigobon, R. (2002). No contagion, only interdependence: measuring stock market comovement. Journal of Finance, 57(5), 2223-2262.

Gallegati, M. (2012). A wavelet-based approach to test for financial market contagion. Computational Statistics \& Data Analysis, 56(11), 3491-3497.

Guesmi, K., Kaabia, O., \& Kazi, I. (2013). Does shift contagion exist between OECD stock markets during the financial crisis? The Journal of Applied Business Research, 29(2), 469-484.

Hemche, O., Jawadi, F., Maliki, S.B., \& Cheffou, A.I. (2016). On the study of contagion in the context of the subprime crisis: A dynamic conditional correlation-multivariate GARCH approach. Economic Modelling, 29 (January), 252-259.

Horta, P., Mendes, C., \& Vieira, I. (2009). Contagion effects of the subprime crisis in the European NYSE Euronext markets. Portuguese Economic Journal, 9(2), 115-140.

Horvath, R., \& Poldaufy, P. (2012). International stock market comovements: What happened during the financial crisis? Global Economy Journal, 12(1), 1-19.

Huang, B.N., Yang, C.W., \& Hu, J.W. (2000). Causality and cointegration of stock markets among the United States, Japan and the South China growth triangle. International Review of Financial Analysis, 9(3), 281-297.

Inci, A.C., Li, H.C. y McCarthy, J. (2011). Financial contagion: A local correlation analysis. Research in International Business and Finance, 25(1), 11-25.

Johansen, S. (1988). Statistical analysis of co-integration vectors. Journal of Economics Dynamics and Control, 12(2-3), 231-254.

Khan, S., \& Park, K.W. (2009). Contagion in the stock markets: The Asian financial crisis revisited. Journal of Asian Economics, 20(5), 561-569.

Koenker, R., \& Bassett, G. (1978). Regression quantiles. Econometrica, 46(1), 33-50.

Liew, V.K. (2004). Which lag length selection criteria should we employ? Economics Bulletin, 3(33), $1-9$.

Longin, F., \& Solnik, B. (1995). Is correlation in international equity returns constant: 1960-1990? Journal of International Money and Finance, 14(1), 3-26.

Longin, F., \& Solnik, B. (2001). Extreme correlation of international equity markets. Journal of Finance, 56(2), 649-676. 
Mathur, I., Gleason, K. C., \& Singh, M. (2002). Contagion effects from the 1994 Mexican Peso crisis: Evidence from Chilean stocks. The Financial Review, 17(1), 17-34.

Mollaha, S., Quoreshib, A.M.M., \& Zafirov, G. (2016). Equity market contagion during global financial and Eurozonecrises: Evidence from a dynamic correlation analysis. Journal of International Financial Markets, Institutions \& Money, 41(March), 151-167.

Naoui, K., Liouane, N., \& Brahim, S. (2010). A dynamic conditional correlation analysis of financial contagion: the case of the subprime credit crisis. International Journal of Economics and Finance, 2(3), 85-96.

Osterwald-Lenum, M. (1992). A note with quantiles of the asymptotic distribution of the máximum likelihood cointegration rank test statistics. Oxford Bulletin Economics and Statistics, 54(3), $461-72$.

Peng, Y., \& Ng, W.L. (2012). Analysing financial contagion and asymmetric market dependence with volatility indices via copulas. Annals of Finance, 8(1), 49-74.

Politis, D.N., \& Romano, J.P. (1994). The stationary bootstrap. Journal of the American Statistical Association, 89(428), 1303-1313.

Rodriguez, J.C. (2007). Measuring financial contagion: A copula approach. Journal of Empirical Finance, 14(3), 401-423.

Serwa, D., \& Bohl, M.T. (2005). Financial contagion vulnerability and resistance: A comparison of European stock markets. Economics Systems, 29(3), 344-362.

Syllignakis, M.N., \& Kouretas, G.P. (2011). Dynamic correlation analysis of financial contagion. Evidence from the Central and Eastern European markets. International Review of Economics and Finance, 20(4), 717-732.

Wang, K.M., \& Nguyen, T.B. (2013). Did China avoid the Asian Flu? The contagion effect test with dynamic correlation coefficients. Quantitative Finance, 13(3) 471-481. 